\title{
Coexistence of Surface Superconducting and Three-Dimensional Topological Dirac States in Semimetal KZnBi
}

\author{
Junseong Song $\odot,{ }^{1,2, *}$ Sunghun Kim, ${ }^{3, *}$ Youngkuk Kim $\odot,{ }^{4, *}$ Huixia Fu, ${ }^{5}$ Jahyun Koo, ${ }^{5}$ Zhen Wang, ${ }^{1}$ Gyubin Lee, ${ }^{3}$ \\ Jouhahn Lee, ${ }^{6}$ Sang Ho Oh๑, ${ }_{1}^{1}$ Joonho Bang, ${ }^{1}$ Taku Matsushita $\odot,{ }^{7}$ Nobuo Wada, ${ }^{7}$ Hiroki Ikegami $\odot,{ }^{8}$ \\ Jonathan D. Denlinger, ${ }^{9}$ Young Hee Lee, ${ }^{1,2}$ Binghai Yan, ${ }^{5, \uparrow}$ Yeongkwan Kim, ${ }^{3,10, \sharp}$ and Sung Wng Kim ${ }^{1,2, \S}$ \\ ${ }^{1}$ Department of Energy Science, Sungkyunkwan University, Suwon 16419, Republic of Korea \\ ${ }^{2}$ Center for Integrated Nanostructure Physics, Institute for Basic Science, Suwon 16419, Republic of Korea \\ ${ }^{3}$ Department of Physics, Korea Advanced Institute of Science and Technology, \\ Daejeon 34141, Republic of Korea \\ ${ }^{4}$ Department of Physics, Sungkyunkwan University, Suwon 16419, Republic of Korea \\ ${ }^{5}$ Department of Condensed Matter Physics, Weizmann Institute of Science, Rehovot 7610001, Israel \\ ${ }^{6}$ Advanced Nano Surface Research Group, Korea Basic Science Institute, \\ Daejeon 34133, Republic of Korea \\ ${ }^{7}$ Department of Physics, Nagoya University, Chikusa-ku, Nagoya 464-8602, Japan \\ ${ }^{8}$ RIKEN Center for Quantum Computing (RQC), RIKEN, Wako, Saitama 351-0198, Japan \\ ${ }^{9}$ Advanced Light Source, Lawrence Berkeley National Laboratory, California 94720, USA \\ ${ }^{10}$ Graduate School of Nanoscience and Technology, Korea Advanced Institute of Science and Technology, \\ Daejeon 34141, Republic of Korea
}

(Received 25 August 2020; revised 13 March 2021; accepted 7 May 2021; published 28 June 2021)

We report the discovery of a new three-dimensional (3D) topological Dirac semimetal (TDS) material $\mathrm{KZnBi}$, coexisting with a naturally formed superconducting state on the surface under ambient pressure. Using photoemission spectroscopy together with first-principles calculations, a 3D Dirac state with linear band dispersion is identified. The characteristic features of massless Dirac fermions are also confirmed by magnetotransport measurements, exhibiting an extremely small cyclotron mass of $m^{*}=0.012 m_{0}$ and a high Fermi velocity of $v_{F}=1.04 \times 10^{6} \mathrm{~m} / \mathrm{s}$. Interestingly, superconductivity occurs below $0.85 \mathrm{~K}$ on the (001) surface, while the bulk remains nonsuperconducting. The captured linear temperature dependence of the upper critical field suggests the possible non-s-wave character of this surface superconductivity. Our discovery serves a distinctive platform to study the interplay between 3D TDS and the superconductivity.

DOI: 10.1103/PhysRevX.11.021065

\section{INTRODUCTION}

The discovery of new topological materials is of great interest in the field of condensed matter physics. Among the various topological materials, three-dimensional (3D) topological Dirac semimetals (TDSs) are distinctive as various topological phases can be induced from TDS [1-4]. For instance, breaking inversion or time-reversal symmetry

\footnotetext{
*These authors contributed equally to this work.

†binghai.yan@weizmann.ac.il

†yeongkwan@kaist.ac.kr

§kimsungwng@skku.edu
}

Published by the American Physical Society under the terms of the Creative Commons Attribution 4.0 International license. Further distribution of this work must maintain attribution to the author(s) and the published article's title, journal citation, and DOI.

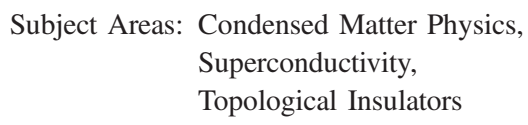

leads to a Weyl semimetal phase with separated Weyl cones [3-5]. Reducing rotational symmetry raises a 3D topological insulator phase with massive bulk Dirac fermions [3-5]. Furthermore, it is believed that 3D TDSs can host topological superconductivity [1,2,6-12]. This interesting possibility has been explored with pressure-, tip-point contact-, and proximity-induced superconductivity in 3D TDS $\mathrm{Cd}_{3} \mathrm{As}_{2}$ [7-11] and bulk superconductivity with Dirac state at the Fermi level $\left(E_{F}\right)$ in Pt-doped $\operatorname{IrTe}_{2}$ [12].

Recently, a variety of hexagonal $A B C$ ternary systems, which have a wide range of spin-orbit coupling (SOC) strengths and spatial symmetries, have been discovered to possess diverse topological states including topological insulators, TDSs, and hourglass fermions [13-16]. Among $A B C$ systems with a layered honeycomb structure, which belong to the space group $P 6_{3} / m m c$ [13-15], potassium (K) stuffed compounds produce a planar honeycomb structure by virtue of the large ionic radius of K [Fig. 1(a)]. The alternative 
(a)

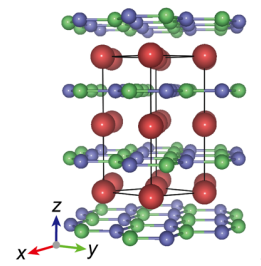

(d)

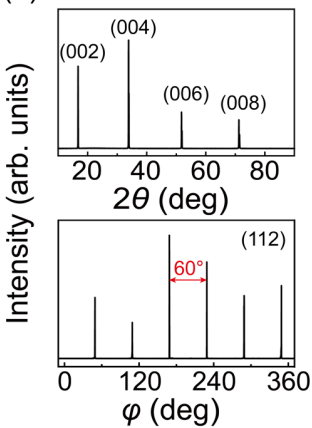

(b)
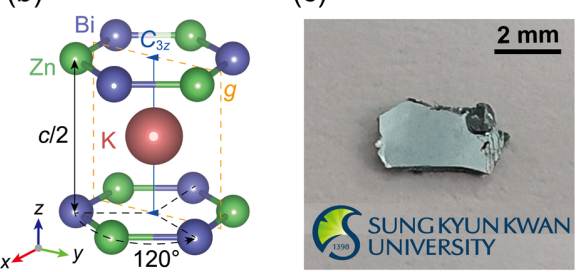

(e)

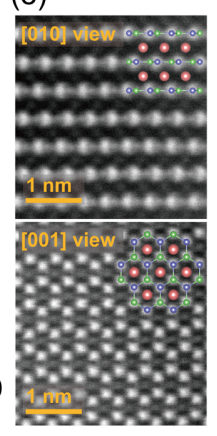

(f)

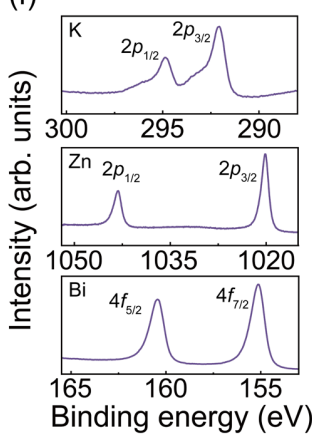

FIG. 1. Crystal structure of KZnBi. (a),(b) Atomic structure of $\mathrm{KZnBi}$, which belongs to the $P 6_{3} / m m c$ space group (a). The solid lines represent a conventional hexagonal unit cell. The $\mathrm{Zn}$ (green) and $\mathrm{Bi}$ (purple) ions form honeycomb layers with an $A B$ stacking order while the $\mathrm{K}$ ion is located at the inversion center. The plane indicated by orange dashed lines $(g)$ denotes the glide mirror plane and the blue solid line shows the principal axis of the threefold rotation symmetry $\left(C_{3 z}\right)$ (b). (c) Image of high-quality $\mathrm{KZnBi}$ single crystal with a few millimeters size. (d) Singlecrystal XRD patterns for the family of (00l) peaks (top) and periodic reflections of (112) plane (bottom). (e) High-angle annular dark-field (HAADF)-STEM images taken along [010] (top) and [001] (bottom) zone axes. (f) XPS spectra of K $2 p$ (top), Zn $2 p$ (middle), and Bi $4 f$ (bottom) orbitals.

stacking of planar honeycomb $B C$ layers and $\mathrm{K}$ ions gives unique spatial symmetries, which include an inversion symmetry centered around the $\mathrm{K}$ ions and a threefold rotational symmetry in the honeycomb [Fig. 1(b)]. This offers an opportunity to realize an exotic topological phase that depends on the combination of $B$ and $C$ elements, which can tune possible band inversion through SOC strength.

In this work, we study $\mathrm{KZnBi}$ that is constructed from planar honeycomb $\mathrm{ZnBi}$ layers, which has not been listed in any recently built databases for topological materials [17-19]. Evidence collected from angle-resolved photoemission spectroscopy (ARPES), first-principles calculations, and magnetotransport measurements of single-crystal $\mathrm{KZnBi}$ verify the existence of 3D Dirac cones. Further, the emergence of surface superconductivity is observed below $0.85 \mathrm{~K}$ under ambient pressure and inert conditions, which coexists with the nonsuperconducting bulk TDS state.

\section{RESULTS AND DISCUSSION}

\section{A. The crystal structure of $\mathrm{KZnBi}$}

A high-quality single crystal of $\mathrm{KZnBi}$ with a shiny emerald color [Fig. 1(c)] was grown by the self-flux

method (see the Appendix). The single crystal was characterized by exclusive $(00 l)$ reflection peaks [top of Fig. 1(d)] and periodic reflections with $60^{\circ}$ [bottom of Fig. 1(d)] from out-of-plane $(2 \theta)$ and in-plane $(\varphi)$ x-ray diffraction (XRD) (see powder XRD in Fig. S1 [20]). Scanning transmission electron microscopy (STEM) was used to confirm the alternative stacking of planar honeycomb ZnBi layers with K ions [Fig. 1(e)]. X-ray photoelectron spectroscopy (XPS) measurements [Fig. 1(f)] revealed that the $\mathrm{K}$ ion is in a monovalent cationic state $\left(\mathrm{K}^{+}\right)$and the honeycomb $\mathrm{ZnBi}$ layer is in a negatively charged state $\left([\mathrm{ZnBi}]^{-}\right)$. One electron transfer to the $\mathrm{ZnBi}$ layer satisfies the Octet rule of valence electron counts [21] for the $s p^{2}$ hybrid orbital bonding between $\mathrm{Zn}$ and $\mathrm{Bi}$ orbitals, forming the planar honeycomb structure (see Appendix and Fig. S1 [20]). In this honeycomb structure, the inversion symmetry, which enforces well-defined parities on the electron wave functions, induces double degeneracy in all bands together with the time-reversal symmetry. In addition, a glide mirror symmetry, $y z$ mirror reflection combined with a translational shift (i.e., a $c / 2$ shift where $c$ is the lattice parameter) along the $z$ axis, leads to a degeneracy at the Brillouin zone (BZ) boundary. Finally, the threefold rotational symmetry around the $z$ axis protects the band crossing along the $k_{z}$ axis and produces the Dirac points.

\section{B. Calculated electronic structure}

In the calculated band structure, a pair of Dirac points (labeled " $D$ ") appear at the $\Gamma-A$ line in the $3 \mathrm{D}$ BZ [Fig. 2(a)]. The bulk bands are nearly two dimensional, but with finite dispersions along the $k_{z}$ direction [Fig. 2(b)]. Without the SOC, the band structure exhibits a full gap (Fig. S2 [20]) between the Zn $s$ conduction bands and Bi $p$ valence bands. As SOC comes in, the top Bi $p$ valence bands ("-" parity, labeled as $\Gamma_{10}^{-}$) are pushed up to cross the lowest Zn $s$ conduction bands ("+" parity, labeled as $\Gamma_{9}^{+}$) along the $\Gamma-A$ direction [Fig. 2(c)]. Along this highsymmetry line, the two crossing bands have different space group representations $\left(\Delta_{7}\right.$ and $\left.\Delta_{9}\right)$ that are distinguished by their threefold rotational symmetry. Therefore, the crossing point can be classed as a symmetry-protected fourfold degenerate Dirac point. This band crossing scenario is similar to that seen in a previously studied TDS, $\mathrm{Na}_{3} \mathrm{Bi}$, which belongs to the same space group [22]. In $\mathrm{KZnBi}$, a pair of Dirac points exist at $k_{z}= \pm 0.6 \pi / c$ along the rotational axis. Near the Dirac point, the band dispersion is rather anisotropic with high in-plane Fermi velocity $\left(\sim 1 \times 10^{6} \mathrm{~m} / \mathrm{s}\right)$ and low out-of-plane velocity $\left(\sim 1 \times 10^{5} \mathrm{~m} / \mathrm{s}\right)$. As the Fermi energy $\left(E_{F}\right)$ shifts down from the Dirac points, two anisotropic pockets appear at the Fermi surface. As shown in Fig. 2(d), two independent pockets touch each other below $-50 \mathrm{meV}$ and form a small pocket embedded inside a large pocket. Therefore, experimental determinations of the Fermi surface topology and 
(a)

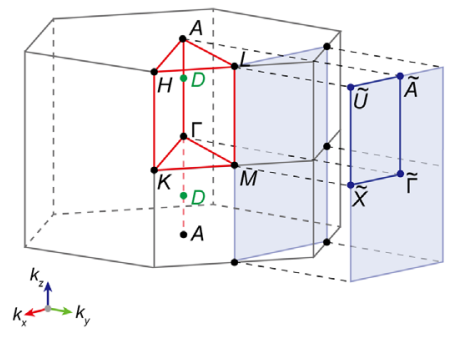

(d)

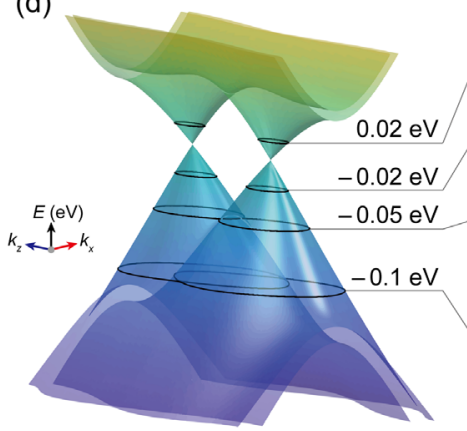

(b)

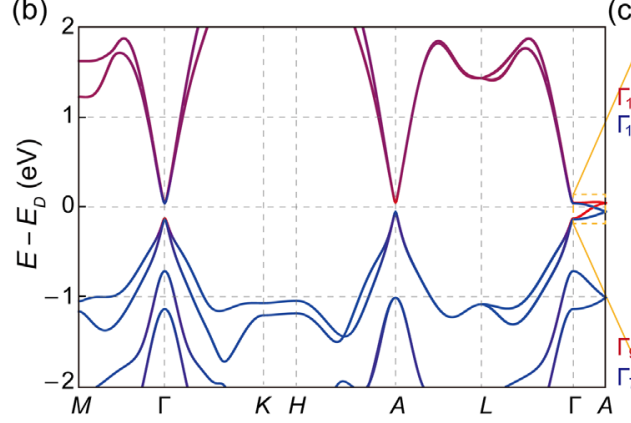

(e)

(f)

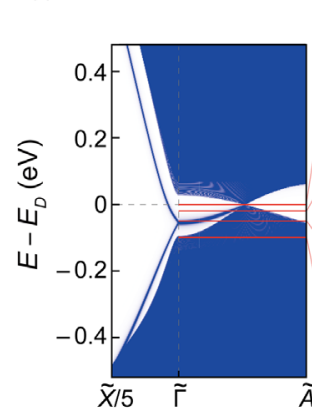

(c)

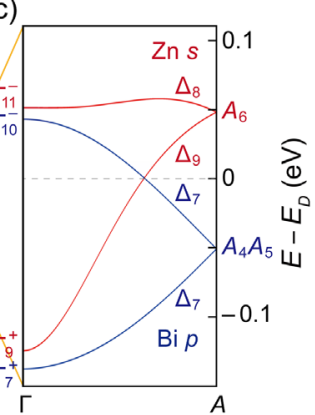

(g)

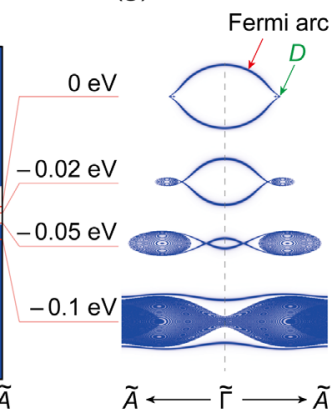

FIG. 2. Calculated band structure of KZnBi. (a) Bulk and (010) projected surface BZ of KZnBi with high symmetric points. Two Dirac points (green dots, labeled " $D$ ") exist along the $\Gamma$ - $A$ line. (b),(c) Calculated band structure of KZnBi along the high symmetry lines (b) and the expanded band structure along $\Gamma-A$ (c). The red and blue represent the $\mathrm{Zn} s$ and $\mathrm{Bi} p$ orbitals, respectively. (d) Dirac bands on the $k_{x}-k_{z}$ plane. (e) Anisotropic isoenergy surfaces of Dirac bands along the $k_{z}$ direction. A small pocket is forming when $E_{F}$ is located below $-50 \mathrm{meV}$. (f) Calculated surface state for (010) plane. (g) Isoenergy surfaces of the surface band structure with Dirac points and Fermi arcs.

carrier density are sensitive to the chemical potential position that varies from sample to sample. In addition, the side surface of the TDS displays topological surface states $[22,23]$. The calculated surface states on the $x z$ plane [(010) surface] are shown in Fig. 2(f). From the band inversion that occurs at $\Gamma$, a pair of Fermi arcs link two Dirac points on the surface by crossing the $k_{z}=0$ plane [Fig. 2(g)].

\section{Direct observation of electronic structure}

ARPES measurements provide direct evidence of the existence of Dirac fermions and prove the 3D nature of the Dirac cone in KZnBi. Band dispersion along the $\Gamma$-K direction $\left(k_{z}=0\right)$ of as-cleaved $\mathrm{KZnBi}$ is shown in Fig. 3(a1) (Fig. S3 [20] for $\Gamma-M$ direction). Compared with the calculated band structure (solid blue line), the $E_{F}$ is pinned on the valance band side, indicating that the sample is slightly hole doped. To confirm the Dirac cone, the $E_{F}$ was tuned by electron doping via in situ deposition of $\mathrm{Rb}$ atoms on the surface of $\mathrm{KZnBi}$. The conduction band clearly appears by a continuous rigid band shift that occurs with the increase in Rb coverage [Figs. 3(a2) and 3(a3)]. Then, to explore the 3D Dirac fermions that are expected to be located between $\Gamma$ and $A$, as marked in Fig. 2(a), the band dispersion from $\Gamma$ to $A$ was traced by tuning the photon energies, which determine the $k_{z}$ positions [ 1 to 6 in Fig. 3(b)]. The detailed band dispersion and stacked momentum distribution curves (MDCs) are plotted in Figs. 3(c) and 3(d), respectively. Both conduction and valance bands show hyperbolic dispersion at $\Gamma$ [Figs. 3(c1) and 3(d1)]. As $k_{z}$ increases, the band gap (indicated by white lines) continuously decreases [Figs. 3(c2) to 3(c3) and $3(\mathrm{~d} 2)$ to $3(\mathrm{~d} 3)]$ before disappearing at $k_{z}=0.6 \pi / c$ [Figs. 3(c4) and 3(d4)]. We note that a linear band dispersion at $k_{z}=0.6 \pi / c$ [Figs. 3(c4) and 3(d4)], clearly demonstrates the existence of Dirac fermions. Furthermore, the observed $k_{z}$ location of the Dirac point agrees well with our $a b$ initio calculation that predicts a pair of Dirac points at $\pm k_{z}=0.6 \pi / c$. As $k_{z}$ increases further to $\pi / c$ [Figs. 3(c5) to 3(c6) and 3(d5) to 3(d6)], the band gap reopens with hyperbolic band dispersion. The detailed dispersions at each $k_{z}$ position are consistent with the calculations given in Fig. 3(e). From the theoretical calculation and experimental verification of bulk Dirac fermions, we thus conclude that the KZnBi is 3D TDS.

\section{Quantum transport measurement}

The quantum transport of 3D Dirac fermions in KZnBi was examined using magnetic fields. Figure 4(a) plots the resistivity $\rho_{x x}$ versus temperature $T$ for a representative 
(a)

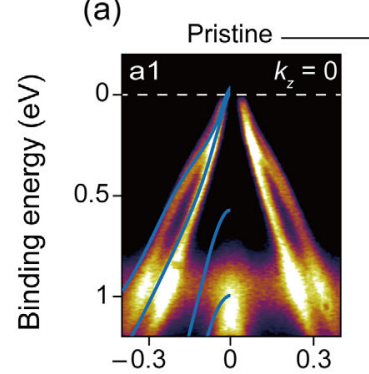

Surface $\mathrm{Rb}$ doping

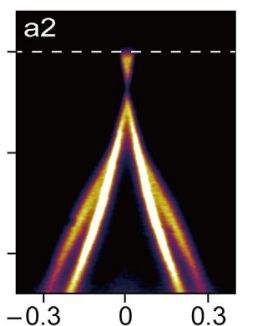

Momentum $k_{y}\left(\AA^{-1}\right)$

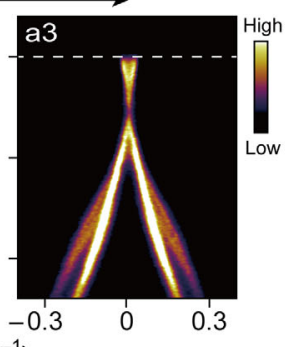

(b)
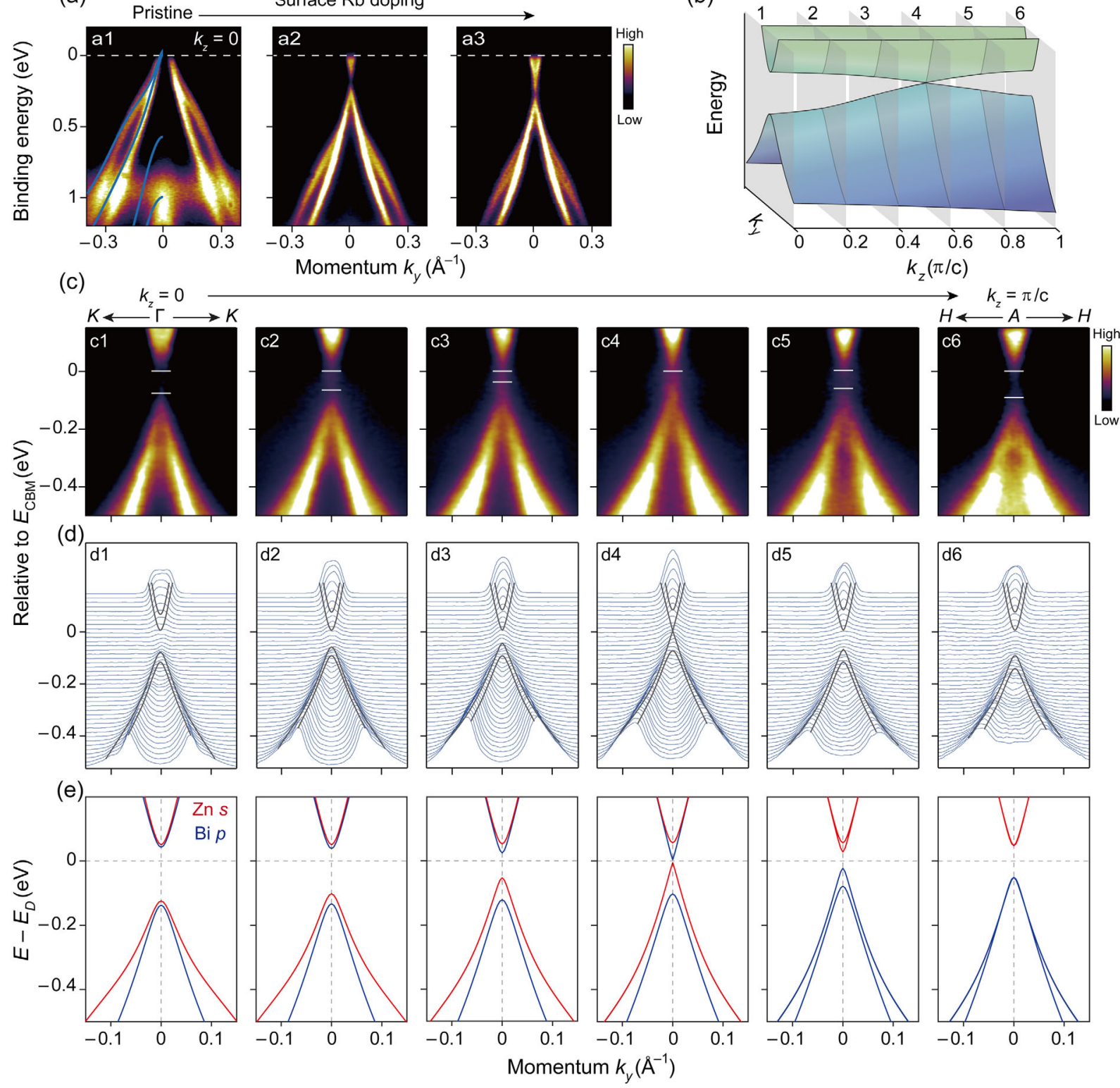

FIG. 3. Direct observation of 3D Dirac fermions from angle-resolved photoemission spectroscopy (ARPES). (a) ARPES spectra of the pristine sample along the $\Gamma$-K direction at $k_{z}=0(\mathrm{a} 1)$. The overlaid blue solid lines show the calculated band structure. $E_{F}$ is rising with surface Rb doping and the conduction band appears [(a2) and (a3)]. (b) Schematic of 3D Dirac cone with slices (gray planes) at different positions of $k_{z}$. (c) Measured band dispersions after surface Rb doping along the $k_{y}$ direction at different $k_{z}$ positions from 0 to $\pi / c$ [(c1) -(c6)]. The $k_{z}$ positions of each panel correspond to those presented in (b) and are numbered accordingly. White lines represent the conduction band minima (CBM) and valance band maxima (VBM). The CBM energy in each panel was set to $0 \mathrm{eV}$ after subtracting the $\mathrm{Rb}$ impurity level. (d) Stacked MDCs of (c). Overlaid gray solid lines are guidelines for the band dispersion. (e) Calculated band dispersions at $k_{z}$ positions corresponding to (c) and (d). The red and blue solid lines represent Zn $s$ and Bi $p$ orbitals, respectively.

sample A1 under a transverse magnetic field $B$ of up to 14 Tesla (T). As the field strength increases, the behavior of $\rho_{x x}(T)$ changes from the positive slope of a semimetal to the negative slope of a semiconductor at $14 \mathrm{~T}$. This is probably due to the band gap opening at the Dirac point induced by Landau level quantization. The Hall resistivity $\rho_{y x}(B)$ has a positive slope proving the hole-doped nature of the material, which is consistent with the ARPES measurements [Figs. 3(a) and S3 [20] ]. A hole concentration $n_{H}$ of $\sim 2.7 \times 10^{18} \mathrm{~cm}^{-3}$ and a mobility $\mu_{H}$ of $1.1 \times 10^{4} \mathrm{~cm}^{2} \mathrm{~V}^{-1} \mathrm{~s}^{-1}$ were obtained at $2 \mathrm{~K}$ for sample $\mathrm{A} 2$ [Fig. 4(b)]. The magnetoresistance (MR), measured at various temperatures, was found to be $\sim 1100 \%$ and $2500 \%$ in respective fields of 9 and $14 \mathrm{~T}$ at $2 \mathrm{~K}$ [Figs. 4(c) and S6 [20] ]. All the measured samples exhibit relatively high mobility $\left(>10^{3} \mathrm{~cm}^{2} \mathrm{~V}^{-1} \mathrm{~s}^{-1}\right)$ and large MR 

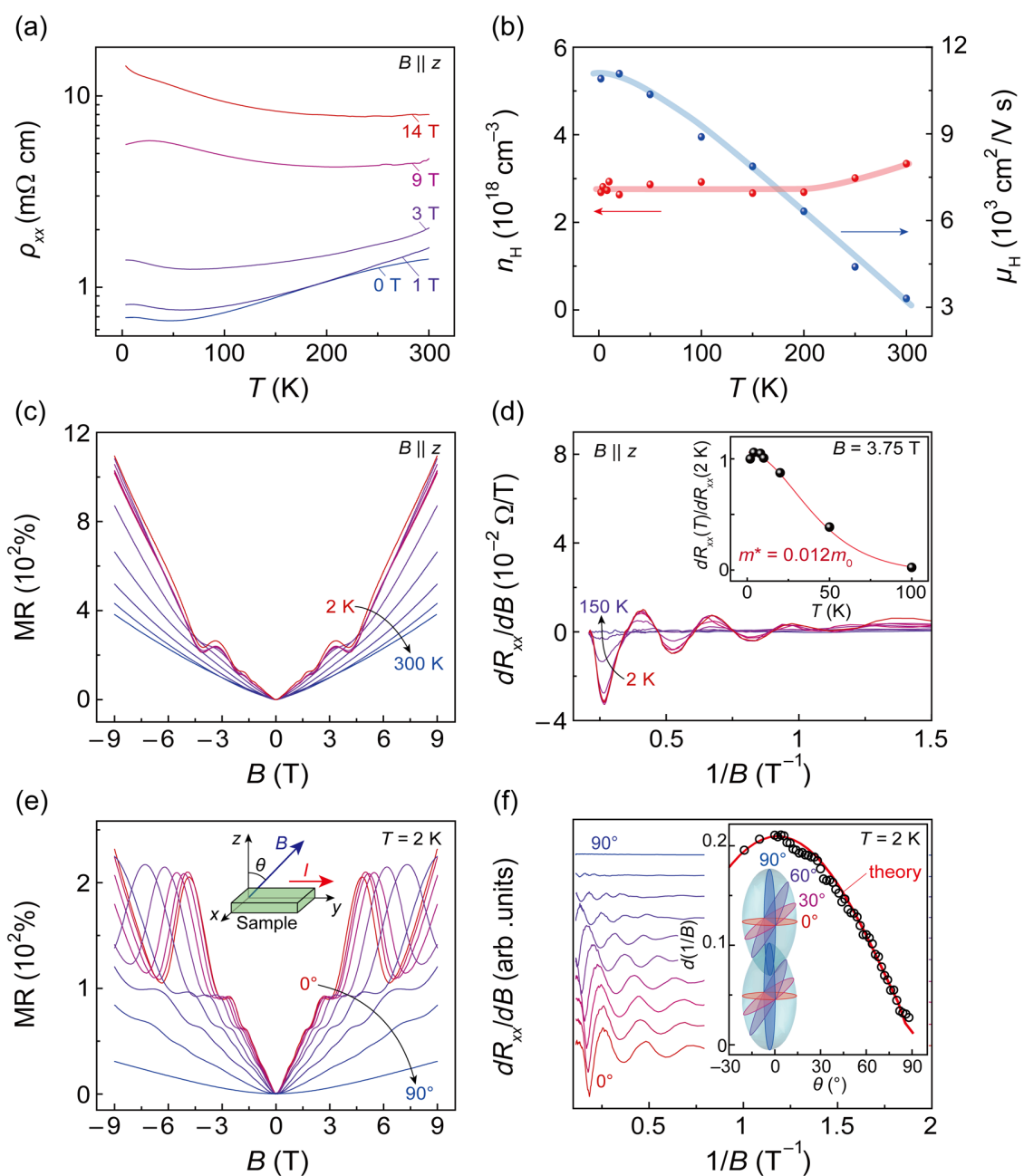

FIG. 4. Quantum transport properties of 3D Dirac fermions. (a) $T$ dependence of electrical resistivity $\rho_{x x}$ under various magnetic fields along the $z$ direction. (b) $T$ dependence of carrier concentration $n_{H}$ (red dots) and mobility $\mu_{H}$ (blue dots). (c) MR under $B$ of up to 9 T at various $T$. SdH oscillations appear at low $T$ and vanish as $T$ increases. (d) $\mathrm{SdH}$ oscillation component $d R_{x x} / d B$ as a function of $1 / B$ at various $T$ from 2 to $150 \mathrm{~K}$, extracted from MRs in (c) by subtracting backgrounds. The inset shows $T$ dependence of the normalized amplitude of the oscillations at $B=3.75 \mathrm{~T}$. The red solid line is fit to the Lifshitz-Kosevich formula. (e),(f) Angle-dependent MRs (e) and SdH oscillations (f) at $2 \mathrm{~K}$. The measurement geometry is illustrated in the inset of (e). The inset of (f) shows that the measured periodicity $d(1 / B)$ (black circles) matches with the theoretically calculated values (red line) for the Dirac band. Schematic illustration shows the cross-sectional Fermi surface area corresponding to the various $B$ field directions, this is shown in Fig. S6(k) [20] with the real size of the Fermi surface in BZ.

$\left(>10^{2} \%\right)$ values at room temperature, demonstrating the transport characteristics of Dirac fermions [24,25]. The properties for all the measured samples are listed in Table S1, Figs. S4 and S5 [20].

From Shubnikov-de Haas (SdH) oscillations [Fig. 4(d)], the Fermi surface size and cyclotron mass of the Dirac fermions were estimated. Analysis of the oscillations of $d R_{x x} / d B$ gives the periodicity $d(1 / B)=0.271 \mathrm{~T}^{-1}$ (frequency, $F=3.69 \mathrm{~T}$ ), which reveals a small cross-sectional area of the Fermi surface $S_{F}=3.5 \times 10^{-4} \AA^{-2}$ and the Fermi momentum $k_{F}=0.11 \AA^{-1}$ along the $k_{x}-k_{y}$ plane (see Appendix). The obtained values are in good agreement with the observed values for Fermi surface size and Fermi momentum from the ARPES measurements (Fig. S3 [20]).
By plotting the normalized amplitude of the $\mathrm{SdH}$ oscillations as a function of $T$ [inset of Fig. 4(d), $d R_{x x}(T) / d R_{x x}(2 \mathrm{~K})$ at $\left.B=3.75 \mathrm{~T}\right)$, it is obtained that an extremely small value for cyclotron mass $m^{*}=0.012$ $m_{0}$, where $m_{0}$ is the bare electron mass, which is the expected characteristics of Dirac fermions. The shape of the Fermi surface is deduced from the angle $(\theta)$ dependent MR and the SdH oscillations [Figs. 4(e) and 4(f)]. Looking at Fig. 4(f), as $B$ rotates from $\theta=0^{\circ}(B \| z)$ to $\theta=90^{\circ}(B \| I)$, the measured periodicity of the $\mathrm{SdH}$ oscillations (black circles in the inset) abruptly decreases. This behavior matches that predicted by the Fermi surface calculations (red line in the inset). Our results consistently indicate an elongated Fermi surface along the $k_{z}$ direction, supporting 
the calculated band structure (for detailed information about the small pocket, see Fig. S6 [20]).

\section{E. Surface superconducting state of $\mathrm{KZnBi}$}

Remarkably, it turns out that the 3D TDS KZnBi exhibits a superconducting transition. Figure 5(a) shows that all the bulk single crystals clearly show zero resistance below the superconducting transition temperature $T_{c}$ of $\sim 0.85 \mathrm{~K}$ (see Fig. S7 [20] for details). However, it should be noted that neither a jump in heat capacity $(C)$ nor a diamagnetic response in ac magnetic susceptibility were observed [inset of Fig. 5(a) and Fig. S8 [20]]. This indicates an extremely small volume fraction of the superconductivity. Specifically, rigorous ac susceptibility measurements suggest that the superconducting volume fraction is less than $1 \%$ (details in Appendix).

For this unusual superconductivity, we rule out the filamentary superconductivity from the result that the superconducting critical current $\left(I_{c}\right)$ below $T_{c}$ does not depend on the volume of the sample [Fig. 5(b)]. Instead, every recorded $I_{c}$ from all the measured samples with different geometries shows a similar current density per unit width $\left(a_{c}=I_{c} / w\right)$, thus supporting surface superconductivity [see Fig. 5(c) and details in Fig. S9 [20] ]. This width dependence of the critical current implies that surface superconductivity most likely emerges on the (001) top surface rather than the lateral surface where a topological Fermi arc surface state is expected. The upper critical fields $\left(H_{c 2}\right)$ measured in parallel (top) and perpendicular (bottom) $B$ directions to the $z$ axis show anisotropy $\left[H_{c 2}(B \perp z) / H_{c 2}(B \| z)\right.$ of $\sim 1.25$ [Fig. 5(d)] and Fig. S7 [20]]. This anisotropy is similar to what is observed in $\mathrm{Sb}_{2} \mathrm{Te}_{3}$ that also hosts surface superconductivity [26]. In addition to its surface nature, it is notable that the $H_{c 2}$ shows a linear $T$ dependence [inset of Fig. 5(e) and details in Fig. S7 [20] ]. The reduced critical field $h^{*}$ deviates from the Werthamer-Helfand-Hohenberg (WHH) curve of an $s$-wave superconductor (black dashed line) in the lower range of $T / T_{c}$ [Fig. 5(c)] [27]. This small but finite deviation suggests a possible non- $s$-wave character of the present surface superconductivity.
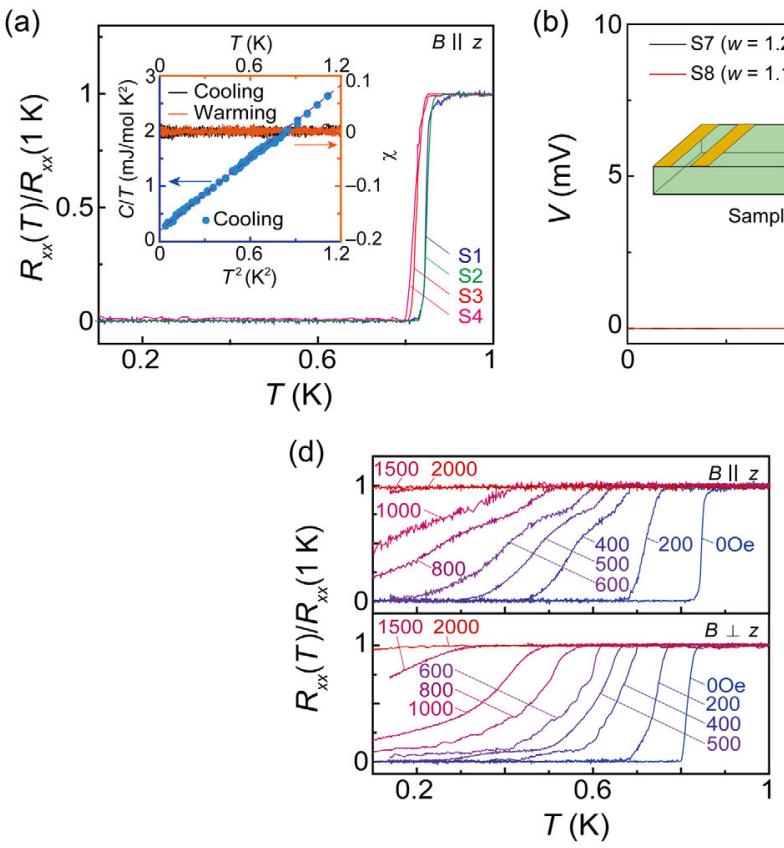
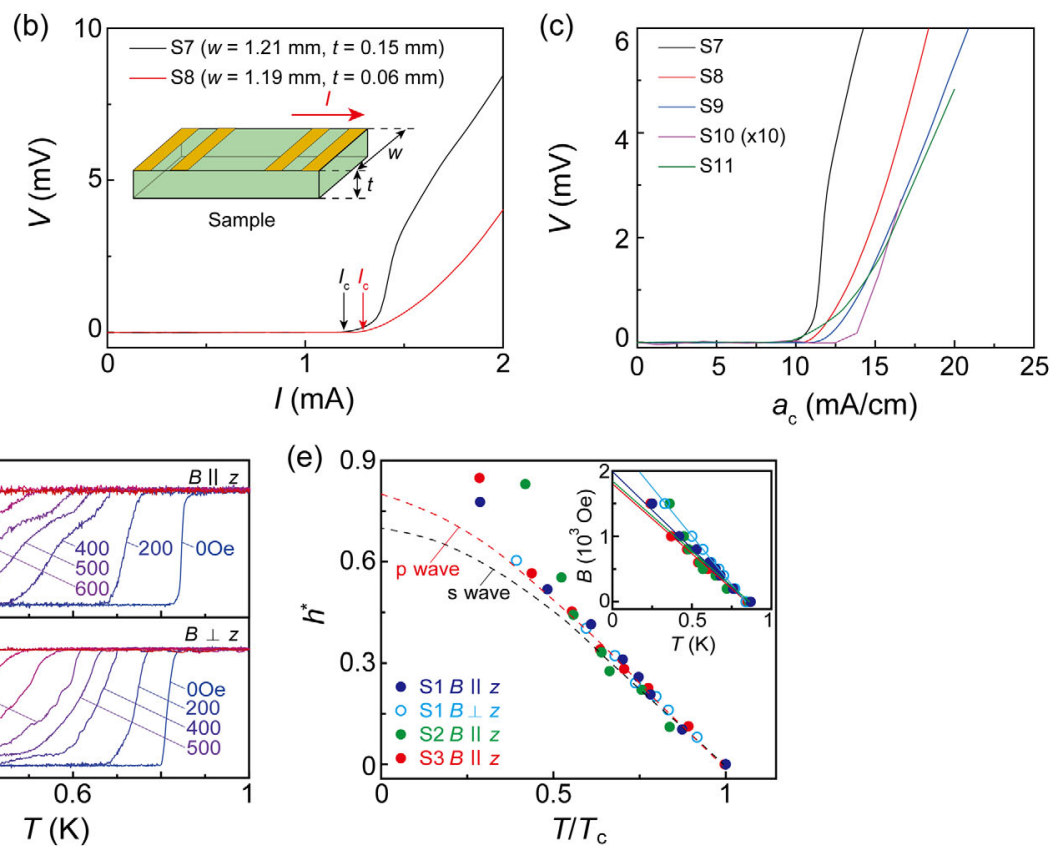

FIG. 5. Surface superconductivity of $\mathrm{KZnBi}$. (a) $T$ dependence of the normalized resistance in single-crystal samples (S1-S4) (Fig. S7 [20]). The inset shows the heat capacity (blue dots), $C / T$, versus $T^{2}$ (left and bottom scales) which can be fitted to a linear function (purple dashed line) and the real part of the ac magnetic susceptibility with decreasing (black solid line) and increasing (orange solid line) $T$ (right and top scales), measured with the single-crystal samples S5 and S6, respectively (see also Fig. S8 [20]). (b) Characteristic current $(I)$-voltage $(V)$ curves of two samples with similar widths $(w)$ and different thicknesses $(t)$ (S7 and S8) at $T=0.1-0.2 \mathrm{~K} . I_{c}$ is indicated by the red and blue arrows for S7 and S8, respectively. S7 is 2.5 times thicker than S8. (c) Critical current density per unit width $\left(a_{c}=I_{c} / w\right)$ for S7-S11 samples. The deviation in $a_{c}$ is smaller than that of the critical current density per unit area $\left(j_{c}=I_{c} / w t\right)$ (Fig. S9 [20]). (d) $T$ dependence of the normalized resistance in S1 under $B$ field parallel (top) and perpendicular (bottom) to the $z$-direction (for S2 and S3, see Fig. S7 [20]). The measurement geometries are illustrated in Fig. S7(g) [20]. (e) $T / T_{c}$ dependence of reduced critical field $\left(h^{*}\right)$ in samples S1-S3 for $B$ parallel (solid dots) and perpendicular (open dots) to the $z$ direction (Appendix). Red and black dashed lines are the WHH curve of $p$-wave and $s$-wave superconductors, respectively. Inset of (e) shows the linear $T$ dependence of $H_{c 2}$ for samples $\mathrm{S} 1-\mathrm{S} 3$. 


\section{CONCLUSIONS}

In summary, we discovered a new 3D TDS, KZnBi with the planar honeycomb lattice of $\mathrm{ZnBi}$, where the Dirac state is protected by threefold rotational and inversion symmetry. Distinguished from previous 3D TDSs, the superconductivity emerges below $0.85 \mathrm{~K}$ without external stimuli, only at the top (001) surface while the bulk remains nonsuperconducting. This unusual surface superconductivity of 3D TDS raises interesting questions that need further investigation, such as looking into the origin of the surface superconductivity as well as examining any interplay that may occur between the superconducting surface and the topological bulk state. In this sense, KZnBi is an excellent candidate for studying the exotic superconductivity in 3D TDS without any external stimuli. This work opens more opportunities for further exploring a new topological material in the class of hexagonal $A B C$ ternary systems with planar honeycomb layered structure.

\section{ACKNOWLEDGMENTS}

We thank J. Chae and J. Park for operating permission for TEM and J. Kim for FIB specimen preparation. This work was supported by the Institute for Basic Science (IBS-R011-D1) and by a National Research Foundation of Korea (NRF) grant funded by the Korean government (Ministry of Science, ICT \& Future Planning) (No. 2015M3D1A1070639, No. 2020R1A4A2002828). The Advanced Light Source is supported by the Office of Basic Energy Sciences of the U.S. DOE under Contract No. DE-AC02-05CH11231.

\section{APPENDIX A: METHODS}

\section{Single-crystal growth}

The single crystal of KZnBi was grown by the self-flux method using alkali metal as the flux. A chunk of $\mathrm{K}$, pulverized $\mathrm{Zn}$ and $\mathrm{Bi}$ in the ratio of $1.1: 1: 1$ were placed in an alumina crucible. The alumina crucible was then double sealed in an evacuated quartz tube under a pressure of $10^{-6}$ Torr to avoid oxidization during the reaction. The double sealed quartz tube containing the alumina crucible was placed in box furnace and the temperature was increased to $630^{\circ} \mathrm{C}$ for $6 \mathrm{~h}$. The sample was kept in $630^{\circ} \mathrm{C}$ for $12 \mathrm{~h}$ then the temperature was slowly decreased down to $300^{\circ} \mathrm{C}$ for a further $100 \mathrm{~h}$. Then, the sample was quenched to room temperature by turning off the furnace. Any additional $\mathrm{K}$ metal is vaporized and reacts with the inner quartz tube during this process. All manipulations for synthesizing the samples were carried out in glove boxes filled with recirculating high-purity $\operatorname{Ar}(99.999 \%)$, this was done to suppress oxygen and moisture because both $\mathrm{K}$ metal and $\mathrm{KZnBi}$ are highly reactive under ambient conditions, following this process water and oxygen concentrations were successfully restricted to less than $0.1 \mathrm{ppm}$.

\section{Structural characterizations}

The crystal structure of KZnBi was investigated by x-ray diffractometry (SmartLab, Rigaku) and scanning transmission electron microscopy (JEM-ARM200F, JEOL). We measured the XRD patterns with the sample under a high-quality $\mathrm{Ar}$ atmosphere in a dome-shaped sample holder, these precautions were taken to prevent any oxidation during the measurements. TEM sample preparation was carried out using a dual-beam focused ion-beam (FIB) slicing and lift-out technique (AURIGA CrossBeam Workstation, Carl Zeiss). In order to prevent the oxidation of samples, an Ar purged glove bag was attached to the entrance of the FIB and TEM chambers during the loading and unloading processes. The XPS measurements were carried out using a micro-X-ray photoelectron spectrometer with a monochromatic $\mathrm{Al} \mathrm{K} \alpha(1486.7 \mathrm{eV}) \mathrm{x}$-ray source. The chamber pressure was maintained below $5.0 \times 10^{-10}$ Torr during the measurements. XPS spectra of a $\mathrm{KZnBi}$ single crystal were obtained by cleaving in an ultrahigh vacuum to prevent oxidation. The shift in the binding energy due to relative surface charging was calibrated as an internal standard using the $\mathrm{C} 1 \mathrm{~s}$ level at $284.6 \mathrm{eV}$.

\section{3. $A b$ initio calculations}

To theoretically simulate the electronic structure of $\mathrm{KZnBi}$, we first performed first-principles calculations using the Vienna $a b$ initio simulation package [28] (VASP) within the framework of density functional theory (DFT). In the DFT calculation, the projector-augmented wave pseudopotential was adopted. Plane waves with a kinetic energy cutoff of $280 \mathrm{eV}$ were used as the basis set. The calculated lattice constants of the periodic unit cell were $a=b=4.68 \AA$ and $c=10.65 \AA$; this is consistent with the experimental results. An $8 \times 8 \times 4$ Monkhorst-Pack $k$ mesh was used for BZ sampling. The modified Becke-Johnson (MBJ) exchange potential [29] in combination with GGA correlation was employed for the exchange-correlation functional to obtain accurate band structures. We constructed the maximally localized Wannier functions [30] of KZnBi from the bulk calculation. Based on the tight-binding parameters of Wannier functions, we designed a semi-infinite open boundary geometry to calculate the surface state with recursive Green's function method [31].

\section{ARPES measurements}

The ARPES measurements were performed at beam line 4.0.3 (MERLIN) of the Advanced Light Source, Lawrence Berkeley National Laboratory. To prevent contamination and oxidation of the $\mathrm{KZnBi}$, sample preparation was performed in an argon atmosphere. The sample was cleaved under a pressure below $5 \times 10^{-11}$ Torr at $15 \mathrm{~K}$ and the 
measurements were carried out under the same conditions. The spectra were accumulated with Scienta R8000 analyzer. Photon energies were varied from $86 \mathrm{eV}\left(k_{z}=0\right)$ to $76 \mathrm{eV}\left(k_{z}=\pi / c\right)$. The overall energy resolution was around $30 \mathrm{meV}$. We determined the $k_{z}$ values based on the $c$-axis lattice constant and the periodicity along the $k_{z}$ direction, obtained from photon energy-dependent measurement with a proper value of inner potential $\left(V_{0}\right)$, by comparing with theoretical calculation. It was found that BZ size along the $k_{z}$ direction is $2 \pi / c \sim 0.6 \AA^{-1}$, this value was arrived at from the experimentally obtained $c$-axis lattice parameter $(10.597 \AA)$. According to the following equation for $k_{z}$,

$$
k_{z}=\sqrt{\frac{2 m\left(E_{k}+V_{0}\right)}{\hbar^{2}}-k_{\|}^{2}},
$$

where $\hbar$ is the Planck constant, $m$ is the electron mass, $E_{k}$ is the kinetic energy of electrons, and $k_{\|}$is the in-plane momentum, we found an appropriate $V_{0}(=9 \mathrm{eV})$ that gives a good match to the experimentally obtained BZ size along the $k_{z}$ direction. After all considerations, the $k_{z}$ values we determined were further verified by comparing the Dirac point position with the theoretically calculated band dispersion. Surface electron doping was carried out by evaporating $\mathrm{Rb}$ on the $\mathrm{KZnBi}$ sample surface at $15 \mathrm{~K}$ using an alkali metal dispenser. Here, we note that there was an unexpected development of spectral weight that originated from the $\mathrm{Rb}$ quantum well state and was observed in addition to the rigid band shift in higher $\mathrm{Rb}$ coverage [Fig. 3(a3)]. As the unexpected spectral weight hinders the band-gap determination, we kept the Rb coverage low enough to avoid an unexpected spectral weight appearance.

\section{Magnetotransport measurements}

All preparation for the measurements was carried out in glove boxes filled with high-purity Ar (99.999\%). To measure the electrical properties of $\mathrm{KZnBi}$, electrical contacts in a six-point probe configuration were made with silver epoxy. Then, Apiezon $\mathrm{N}$ grease was coated onto the sample surfaces to prevent oxidation of the samples during measurement. The measurements were conducted using a physical property measurement system (PPMS DynaCool, Quantum Design). The MR and Hall measurements were performed at temperatures $T$ of 2, 4, 8, 10, 20, $50,100,150,200,250,300 \mathrm{~K}$. The angle-resolved MR measurements were performed at $T=2 \mathrm{~K}$ and with $\theta$ between $-20^{\circ}$ to $90^{\circ}$ (moving in intervals of $10^{\circ}$ ) using the horizontal sample rotator option that is integrated into the DynaCool system. The rotator motor was calibrated according to the angle-dependent resistance of the samples at a magnetic field of 9 T [Figs. S6(f) and S6(g) [20] ]. Lowtemperature measurements below $2 \mathrm{~K}$ were carried out using the dilution refrigerator option that is integrated into the DynaCool system.

\section{Heat capacity measurements}

Specific heat measurements were also performed with the DynaCool PPMS using the dilution refrigerator option. In the low-temperature region, fitting a linear function to $C / T$ versus $T^{2}$ that follows the Debye-Sommerfeld equation, $C / T=\gamma+\beta T^{2}$, allows us to clearly separate the electron $(\gamma)$ and phonon $(\beta)$ contributions to heat capacity.

\section{7. ac magnetic susceptibility measurements}

To investigate the ac susceptibility, measurements to record the change in mutual inductance between the primary and secondary coils at $120 \mathrm{~Hz}$ in the dilution refrigerator were carried out. An ac current $\left(I_{\mathrm{ac}}\right)$ was applied to the primary coil; this induces an ac magnetic field $\left(B_{\mathrm{ac}}\right)$. Then, this ac magnetic field induces an ac voltage $\left(V_{\text {ac }}\right)$ in the secondary coil where we measure the ac susceptibility. From these measurements the $B_{\text {ac }}$ was found to be $33 \mathrm{mG}$ [Figs. S8(e), and S8(f) [20]] and $11 \mathrm{mG}$ [Fig. S8(g) [20]]. In our homemade setup, the ac magnetic field was produced by a small superconducting solenoid coil surrounding the sample in the dilution refrigerator; as such, an ultralow magnetic field was inevitable, giving a sensitivity of superconducting volume fraction of less than $1 \%$. The absolute value of $\chi$ was calibrated from a piece of perfectly diamagnetic $\mathrm{Zn}$ metal.

\section{Chemical bonding nature}

The binding energy of the $\mathrm{K} 2 p_{3 / 2}$ peak $(292.2 \mathrm{eV})$ obtained from the XPS measurements [Fig. 1(f)], located in the $\mathrm{K}^{+}$range $(292-29 \mathrm{eV})$, reveals that the potassium in $\mathrm{KZnBi}$ is cationic in nature with a $\mathrm{K}^{+}$ionic state. The binding energy of the $\mathrm{Zn} 2 p_{3 / 2}(1020.7 \mathrm{eV})$ and $\mathrm{Bi} 4 f_{7 / 2}$ $(155.4 \mathrm{eV})$ in $\mathrm{KZnBi}$ are lower than in their respective metallic states of $\mathrm{Zn} 2 p_{3 / 2}(1021.8 \mathrm{eV})$ and $\mathrm{Bi} 4 f_{7 / 2}$ $(157 \mathrm{eV})$. This indicates that the honeycomb $\mathrm{ZnBi}$ layer is in an anionic state $[\mathrm{ZnBi}]^{-}$. From the XPS measurement data, we note that one electron transfer from $\mathrm{K}$ to $\mathrm{ZnBi}$ layer per unit formula occurs in $\mathrm{KZnBi}$, consists of cationic $\mathrm{K}^{+}$ions and anionic $[\mathrm{ZnBi}]^{-}$layers. This electron transfer allows us to satisfy the Octet rules for the $[\mathrm{ZnBi}]^{-}$layers and form the planar honeycomb layer with $s p^{2}$ hybrid orbital bonding (Fig. S1 [20]).

\section{Fitting the SdH oscillation}

To calculate $m^{*}$, we fit the $T$ dependence of the $\mathrm{SdH}$ amplitudes to the Lifshiz-Kosevich expression,

$$
\frac{\Delta \rho_{x x}(T, B)}{\rho_{x x}(T=2 \mathrm{~K}, B)}=e^{-\Lambda_{D}} \frac{\Lambda}{\sinh (\Lambda)},
$$

where $\Lambda=2 \pi^{2} k_{B} T / \beta, \Lambda_{D}=2 \pi^{2} k_{B} T_{D} / \beta, \beta=e h B / 2 \pi m^{*}$, $k_{B}$ is Boltzmann's constant, $e$ is the charge of an electron, and $T_{D}=h / 4 \pi^{2} \tau_{Q} k_{B}$ is the fitting parameter. 
From the $\mathrm{SdH}$ periodicity $d(1 / B)$, we can calculate the cross-sectional area of the Fermi surface $\left(S_{F}\right)$ using the Onsager relationship

$$
F=\frac{1}{d(1 / B)}=\frac{\hbar}{2 \pi e} S_{F},
$$

where $\hbar$ is the reduced Plank constant. Since KZnBi has a circular cross-sectional Fermi surface, as confirmed by the ARPES study [Fig. S3(c) [20] ], the Fermi wave vector $\left(k_{F}\right)$ can be calculated using the area formula for a circle $\left(S_{F}=\pi k_{F}^{2}\right)$. From $k_{F}$ and $m^{*}$, we calculate the Fermi velocity $v_{F}=\hbar k_{F} / m^{*}$ and the Fermi energy $E_{F}=\hbar v_{F} k_{F}$. (See Table S1 [20] for all samples.)

\section{Reduced critical field}

The reduced critical field $\left(h^{*}\right)$ is expressed as

$$
h^{*}\left(T / T_{c}\right)=-\frac{H_{c 2}}{\left[d H_{c 2} / d\left(T / T_{c}\right)\right]_{T=T_{c}}},
$$

where $H_{c 2}$ is the upper critical field. $H_{c 2}$ is normalized according to the superconducting transition temperature $T_{c}$ to give a relation between $h^{*}$ and $T / T_{c}$. This equation is from Werthamer-Helfand-Hohenberg (WHH) theory and allows the identification of superconductivity.

[1] Z. K. Liu et al., Discovery of a Three-Dimensional Topological Dirac Semimetal, $\mathrm{Na}_{3} \mathrm{Bi}$, Science 343, 864 (2014).

[2] Z. K. Liu et al., A Stable Three-Dimensional Topological Dirac Semimetal $\mathrm{Cd}_{3} \mathrm{As}_{2}$, Nat. Mater. 13, 677 (2014).

[3] Z. Wang, Y. Sun, X. Q. Chen, C. Franchini, G. Xu, H. Weng, X. Dai, and Z. Fang, Dirac Semimetal and Topological Phase Transition in $A_{3} \mathrm{Bi}(A=\mathrm{Na}, K, R b)$, Phys. Rev. B 85, 195320 (2012).

[4] Z. Wang, H. Weng, Q. Wu, X. Dai, and Z. Fang, ThreeDimensional Dirac Semimetal and Quantum Transport in $\mathrm{Cd}_{3} \mathrm{As}_{2}$, Phys. Rev. B 88, 125427 (2013).

[5] J. Xiao and B. Yan, First-Principles Calculations for Topological Quantum Materials, Nat. Rev. Phys. 3, 283 (2021).

[6] S. Kobayashi and M. Sato, Topological Superconductivity in Dirac Semimetals, Phys. Rev. Lett. 115, 187001 (2015).

[7] C. Huang et al., Proximity-Induced Surface Superconductivity in Dirac Semimetal $\mathrm{Cd}_{3} \mathrm{As}_{2}$, Nat. Commun. 10, 2217 (2019).

[8] C.-Z. Li, A.-Q. Wang, C. Li, W.-Z. Zheng, A. Brinkman, D.-P. Yu, and Z.-M. Liao, Fermi-Arc Supercurrent Oscillations in Dirac Semimetal Josephson Junctions, Nat. Commun. 11, 1150 (2020).

[9] L. He, Y. Jia, S. Zhang, X. Hong, C. Jin, and S. Li, PressureInduced Superconductivity in the Three-Dimensional Topological Dirac Semimetal $\mathrm{Cd}_{3} \mathrm{As}_{2}$, npj Quantum Mater. 1, 16014 (2016).
[10] L. Aggarwal, A. Gaurav, G. S. Thakur, Z. Haque, A. K. Ganguli, and G. Sheet, Unconventional Superconductivity at Mesoscopic Point Contacts on the 3D Dirac Semimetal $\mathrm{Cd}_{3} \mathrm{As}_{2}$, Nat. Mater. 15, 32 (2016).

[11] H. Wang, H. Wang, H. Liu, H. Lu, W. Yang, S. Jia, X.-J. Liu, X. C. Xie, J. Wei, and J. Wang, Observation of Superconductivity Induced by a Point Contact on 3D Dirac Semimetal $\mathrm{Cd}_{3} \mathrm{As}_{2}$ Crystals, Nat. Mater. 15, 38 (2016).

[12] F. Fei et al., Band Structure Perfection and Superconductivity in Type-II Dirac Semimetal $\mathrm{Ir}_{1-x} \mathrm{Pt}_{x} \mathrm{Te}_{2}$, Adv. Mater. 30, 1801556 (2018).

[13] X. Zhang, Q. Liu, Q. Xu, X. Dai, and A. Zunger, Topological Insulators versus Topological Dirac Semimetals in Honeycomb Compounds, J. Am. Chem. Soc. 140, 13687 (2018).

[14] H.-J. Zhang, S. Chadov, L. Muchler, B. Yan, X. L. Qi, J. Kubler, S. C. Zhang, and C. Felser, Topological Insulators in Ternary Compounds with a Honeycomb Lattice, Phys. Rev. Lett. 106, 156402 (2011).

[15] Z. Wang, A. Alexandradinata, R. J. Cava, and B. A. Bernevig, Hourglass Fermions, Nature (London) 532, 189 (2016).

[16] H. Gao, Y. Kim, J. W. F. Venderbos, C. L. Kane, E. J. Mele, A. M. Rappe, and W. Ren, Dirac-Weyl Semimetal: Coexistence of Dirac and Weyl Fermions in Polar Hexagonal ABC Crystals, Phys. Rev. Lett. 121, 106404 (2018).

[17] T. Zhang, Y. Jiang, Z. Song, H. Huang, Y. He, Z. Fang, H. Weng, and C. Fang, Catalogue of Topological Electronic Materials, Nature (London) 566, 475 (2019).

[18] M. G. Vergniory, L. Elcoro, C. Felser, N. Regnault, B. A. Bernevig, and Z. Wang, A complete Catalogue of HighQuality Topological Materials, Nature (London) 566, 480 (2019).

[19] F. Tang, H. C. Po, A. Vishwanath, and X. Wan, Comprehensive Search for Topological Materials Using Symmetry Indicators, Nature (London) 566, 486 (2019).

[20] See Supplemental Material at http://link.aps.org/ supplemental/10.1103/PhysRevX.11.021065 for Figs. S1S9 and Table S1.

[21] G. A. Papoian and R. Hoffmann, Hypervalent Bonding in One, Two, and Three Dimensions: Extending the ZintlKlemm Concept to Nonclassical Electron-Rich Networks, Angew. Chem. Int. Ed. 39, 2408 (2010).

[22] Z. Wang, Y. Sun, X. Q. Chen, C. Franchini, G. Xu, H. Weng, X. Dai, and Z. Fang, Dirac Semimetal and Topological Phase Transition in $A_{3} \mathrm{Bi}(A=\mathrm{Na}, K, R b)$, Phys. Rev. B 85, 195320 (2012).

[23] Z. Wang, H. Weng, Q. Wu, X. Dai, and Z. Fang, ThreeDimensional Dirac Semimetal and Quantum Transport in $\mathrm{Cd}_{3} \mathrm{As}_{2}$, Phys. Rev. B 88, 125427 (2013).

[24] T. Liang, Q. Gibson, M. N. Ali, M. Liu, R. J. Cava, and N. P. Ong, Ultrahigh Mobility and Giant Magnetoresistance in the Dirac Semimetal $\mathrm{Cd}_{3} \mathrm{As}_{2}$, Nat. Mater. 14, 280 (2015).

[25] J. Xiong, S. K. Kushwaha, T. Liang, J. W. Krizan, M. Hirschberger, W. Wang, R. J. Cava, and N. P. Ong, Evidence for the Chiral Anomaly in the Dirac Semimetal $\mathrm{Na}_{3} \mathrm{Bi}$, Science 350, 413 (2015).

[26] L. Zhao, H. Deng, I. Korzhovska, M. Begliarbekov, Z. Chen, E. Andrade, E. Rosenthal, A. Pasupathy, V. Oganesyan, and 
L. Krusin-Elbaum Emergent Surface Superconductivity in the Topological Insulator $\mathrm{Sb}_{2} \mathrm{Te}_{3}$, Nat. Commun. 6, 8279 (2015).

[27] E. Helfand and N. R. Werthamer, Temperature and Purity Dependence of the Superconducting Critical Field, $H_{c 2}$. II, Phys. Rev. 147, 288 (1966).

[28] G. Kresse and J. Furthmüller, Effictivent Iterative Schemes for Ab Initio Total-Energy Calculations Using a PlaneWave Basis Set, Phys. Rev. B 54, 11169 (1996).
[29] F. Tran and P. Blaha, Accurate Band Gaps of Semiconductors and Insulators with a Semilocal Exchange-Correlation Potential, Phys. Rev. Lett. 102, 226401 (2009).

[30] I. Souza, N. Marzari, and D. Vanderbilt, Maximally Localized Wannier Functions for Entangled Energy Bands, Phys. Rev. B 65, 035109 (2001).

[31] M. P. L. Sancho, J. M. L. Sancho, and J. Rubio, Highly Convergent Schemes for the Calculation of Bulk and Surface Green Functions, J. Phys. F 15, 851 (1985). 